\title{
Análisis de la laparoscopía diagnóstica y estudios de imagen en el manejo del dolor en fosa ilíaca derecha de etiología incierta
}

\author{
Andrea Sosa*, Patricia Paredes ${ }^{\dagger}$, Gabriela Larrosa ${ }^{\ddagger}$, \\ Andrés Guastavino§, Fabio Maderał, Agustín Pérez
}

\section{Resumen}

Introducción: el dolor en la fosa ilíaca derecha (FID) es un motivo de consulta habitual en los servicios de urgencias. El principal desafío es diferenciar si es un cuadro quirúrgico o no.

Objetivo: conocer qué rol ocupa la laparoscopía diagnóstica (LD), la ecografía de abdomen y la tomografía computada (TC) en el algoritmo de estudio de los pacientes con dolor en FID con diagnóstico incierto en Uruguay.

Método: estudio observacional, descriptivo, prospectivo, multicéntrico, sobre pacientes que consultaron por dolor en FID desde el $1^{\circ}$ de junio al 31 de agosto de 2017 en centros asistenciales de Uruguay. Se recolectaron los siguientes datos en una planilla electrónica: sexo, edad, sintomatología, hallazgos físicos, exámenes solicitados, conducta tomada, hallazgos intraoperatorios, complicaciones.

Resultados: se completaron 159 planillas. Luego de una valoración primaria se planteó duda diagnóstica en 69 (43,4\%) pacientes. La TC se solicitó en 29 (42\%) pacientes. Se realizó TC al 78\% de los mayores de 50 años versus 18\% entre los de 15 y 29 años. Del total, $13(44,8 \%)$ se informaron como normales, confirmando en el intraoperatorio cuatro $(30,7 \%)$ apendicitis agudas. Se realizaron 29 LD en los casos con duda diagnóstica, dos tercios fueron en pacientes de 15 a 29 años. En 22 (76\%) casos se arribó a un diagnóstico. No hubo complicaciones.

Conclusiones: la LD permitió realizar diagnóstico y tratamiento en la mayoría de los casos. La sensibilidad de la TC fue baja en comparación con datos internacionales. El algoritmo diagnóstico debe ser individualizado, basado en protocolos adaptados a los recursos de cada institución.

Palabras clave: Dolor en fosa ilíaca derecha

Apendicitis aguda

Laparoscopía

Diagnóstico por imagen

Key words: $\quad$ Right iliac fossa pain

Acute appendicitis

Laparoscopy

Diagnostic imaging

\footnotetext{
* Residente Cirugía General. Hospital de Minas. Clínica Quirúrgica 3. Facultad de Medicina, Uruguay.

† Residente Cirugía General. Hospital Pasteur. Clínica Quirúrgica 1. Facultad de Medicina, Uruguay.

‡ Residente Cirugía General. Hospital Militar. Clínica Quirúrgica B. Facultad de Medicina, Uruguay.

$\S$ Residente Cirugía General. Sanatorio Casa de Galicia. Clínica Quirúrgica A. Facultad de Medicina, Uruguay.

‡ Residente Cirugía General. Hospital Militar. Clínica Quirúrgica B. Facultad de Medicina, Uruguay.

II Residente Cirugía General. Asociación Española. Clínica Quirúrgica F. Facultad de Medicina, Uruguay.

Los autores declaran no tener conflictos de intereses.

Correspondencia: Andrea Sosa. Héctor Leis 1133, CP 30.000. Minas, Uruguay. Correo electrónico: asosaestela@hotmail.com

Recibido: $14 / 9 / 18$

Aprobado: $7 / 1 / 19$
} 


\section{Introducción}

Las consultas por cuadros de dolor abdominal agudos son comunes en los servicios de urgencia, siendo el dolor en fosa ilíaca derecha (FID) un motivo de consulta habitual. El principal desafío es diferenciar si es un cuadro de resolución quirúrgica o no. El dolor abdominal agudo de causa inespecífica se define como aquel de menos de siete días de duración donde el diagnóstico permanece incierto luego de realizar el examen físico y estudios diagnósticos iniciales ${ }^{(1,2)}$.

Desde que la laparoscopía se introdujo en el campo de la cirugía general muchos cirujanos la han empleado para el diagnóstico y tratamiento de pacientes con abdomen agudo, con particular interés cuando no está clara la etiología del cuadro ${ }^{(1)}$. La laparoscopía diagnóstica (LD) se realiza bajo anestesia general, su precisión diagnóstica en pacientes con dolor abdominal agudo es alta, oscila entre $90 \%$ y $100 \%$ y evita laparotomías innecesarias $^{(2)}$. También se ha propuesto la utilización de la tomografía computada (TC) de abdomen y pelvis como un complemento particularmente útil en la evaluación de pacientes con dolor abdominal inespecífico ${ }^{(2)}$.

El diferente uso de estas herramientas diagnósticas y la falta de protocolos en distintos centros asistenciales de Uruguay motivaron la realización de este trabajo. El objetivo es conocer qué rol ocupa la LD en diferentes centros docente asistenciales (públicos y privados) de Uruguay en el algoritmo de estudio de los pacientes con dolor en FID con diagnóstico incierto, así como analizar el rol de los estudios de imagen como la ecografía de abdomen y la TC.

\section{Material y método}

Se realizó un estudio observacional, descriptivo, prospectivo, multicéntrico, sobre pacientes que consultaron por dolor en FID desde el $1^{\circ}$ de junio al 31 de agosto de 2017. La población seleccionada fue la de pacientes mayores de 15 años que consultaron en servicios de emergencia de distintos centros asistenciales del país: hospitales Pasteur, Maciel, Clínicas, Policial y Militar, sanatorios Casa de Galicia y Asociación Española. Hospitales departamentales de Paysandú, Salto y Minas.

Mediante el uso de una planilla diseñada en formato electrónico, residentes de cirugía general de primer a cuarto año que participaron del trabajo, recabaron los siguientes datos: sexo, edad, sintomatología, hallazgos físicos, exámenes solicitados de laboratorio o de imagen, conducta tomada, hallazgos intraoperatorios y complicaciones.

Análisis estadístico: los resultados fueron resumidos en frecuencias absolutas y relativas porcentuales. Se es-
Tabla 1. Características del dolor y síntomas acompañantes en los casos con duda diagnóstica.

\begin{tabular}{lc}
\hline Características del dolor & Número (\%) n: 69 \\
\hline Dolor en fosa ilíaca derecha & $31(44,9)$ \\
Dolor en hemiabdomen inferior & $21(30,4)$ \\
Esquema de Murphy & $10(14,5)$ \\
Dolor en flanco derecho & $6(8,7)$ \\
Dolor difuso & $1(1,4)$ \\
\hline Síntomas acompañantes & $35(50,7)$ \\
\hline Náuseas y vómitos & $14(20,3)$ \\
Anorexia & $11(16,0)$ \\
Fiebre & $10(14,5)$ \\
Síntomas urinarios & $9(13,0)$ \\
Deposiciones líquidas & \\
\hline
\end{tabular}

timaron los intervalos para las proporciones de sensibilidad, especificidad y valores predictivos con una confianza de 95\%. El análisis se realizó con los programas Excel y Epidat 3.1 para Windows.

\section{Resultados}

En el período del $1^{\circ}$ de junio al 31 de agosto se completaron 159 planillas. Con respecto al sexo, fueron 75 (47\%) hombres y 84 (53\%) mujeres. Edad media de 30 años (rango de 15 a 82 años), con pico máximo a los 17 y 18 años de edad. Los planteos diagnósticos clínicos primarios fueron: duda diagnóstica en $69(43,4 \%)$ pacientes, apendicitis aguda en $68(42,8 \%)$, patología ginecológica en $10(6,3 \%)$, otros diagnósticos en 12 $(7,5 \%)$.

\section{Dolor abdominal en FID con duda diagnóstica}

Luego de una valoración primaria mediante anamnesis, examen físico y paraclínica básica, se planteó duda diagnóstica en $69(43,4 \%)$ pacientes, $46(66,6 \%)$ mujeres y $23(33,4 \%)$ hombres.

El dolor se localizó de inicio en FID o en hemiabdomen inferior en 52 pacientes $(75,4 \%)$. Náuseas y vómitos fueron los síntomas acompañantes más frecuentes, seguidos por anorexia, fiebre, síntomas urinarios y digestivos bajos (tabla 1). Durante el examen físico 54 pacientes $(78,2 \%)$ presentaron dolor a la compresión de FID, solo $9(13 \%)$ presentaron defensa a dicho nivel (tabla 2). 
Tabla 2. Hallazgos durante la exploración física en los casos con duda diagnóstica.

\begin{tabular}{lc}
\hline Hallazgos más frecuentes al examen físico & Número (\%) n: 69 \\
\hline Dolor a la compresión de FID & $54(78,2)$ \\
Dolor espontáneo en FID & $18(26,1)$ \\
Dolor a la decompresión de FID & $13(18,8)$ \\
Defensa en FID & $9(13,0)$ \\
\hline
\end{tabular}

FID: fosa ilíaca derecha

Con respecto a los estudios de imagen, se solicitó ecografía de abdomen a 43 pacientes $(62 \%)$ del total con planteo de duda diagnóstica. En la tabla 3 se compara el informe ecográfico de estos pacientes con el diagnóstico operatorio positivo para apendicitis aguda.

Se solicitó un total de 29 TC (42\%) de abdomen/pelvis. En la figura 1 se representa la relación entre la solicitud o no de TC, según el rango de edad de los pacientes. Del total, $16(55,2 \%)$ fueron diagnósticas: 14 informaron apendicitis aguda y todos fueron intervenidos confirmando el diagnóstico. En dos casos el informe tomográfico derivó en la consulta a otra especialidad. Los restantes 13 casos (44,8\%) se informaron como normales. De estos, a 8 pacientes $(61,5 \%)$ se les realizó una LD confirmando en el intraoperatorio 4 casos $(30,7 \%)$ de apendicitis aguda, de las cuales una presentó un plastrón apendicular abscedado (figura 2).

A 40 pacientes (58\%) con duda diagnóstica no se les solicitó TC, y en 12 pacientes $(17,4 \%)$ no se solicitó ningún estudio de imagen (ni ecografía, ni TC). Los mo- tivos señalados para no solicitar la TC fueron: $26(65 \%)$ no la consideraron necesaria, $9(22 \%)$ no especificaron, 4 (10\%) porque demoraba, $1(3 \%)$ no tenía disponibilidad de tomógrafo. De los 40 pacientes sin TC, a 21 (52,5\%) se les realizó LD: en 11 casos $(52,5 \%)$ se diagnosticó apendicitis aguda, $6(28,5 \%)$ fueron en blanco, 4 (19\%) patología ginecológica. A los 19 restantes (47,5\%) se les otorgó alta, observación o derivaron a otra especialidad. En un solo caso se decidió realizar apendicetomía convencional sin otros estudios.

De los 69 pacientes con duda diagnóstica, se realizaron $29 \mathrm{LD}(42 \%$ ); como se mencionó previamente, ocho pacientes tenían TC informada normal. En la figura $3 \mathrm{se}$ representa la realización o no de $\mathrm{LD}$, según rango de edad. Del total, 15 (51,2\%) diagnosticaron apendicitis aguda, $7(24,1 \%)$ fueron en blanco y $7(24,1 \%)$ otros diagnósticos (figura 4). No hubo complicaciones relacionadas con el procedimiento.

Se diagnosticaron $30(43,5 \%)$ apendicitis agudas de un total de 69 pacientes con duda diagnóstica al inicio del cuadro clínico.

\section{Diagnóstico primario de apendicitis aguda}

Corresponden al sexo masculino 47 pacientes (69\%). El esquema de Murphy estuvo presente en 37 casos $(54,4 \%)$, seguido del dolor de inicio en FID en 22 pacientes $(32,3 \%)$ (tabla 4). Náuseas, vómitos y anorexia fueron los síntomas acompañantes predominantes. En la tabla 5 se representan los hallazgos físicos más frecuentes.

A ninguno de los 68 pacientes se le solicitó TC de abdomen, en 56 casos $(82,3 \%)$, el médico no la consideró necesaria, solo en dos (3\%) porque demoraba su realización. A 55 pacientes $(80,8 \%)$ se les realizó abordaje la-

Tabla 3. Comparación del informe ecográfico con el diagnóstico operatorio de apendicitis aguda.

\begin{tabular}{lccc}
\hline Informe ecográfico & Número $(\%) n: 43$ & Operados & Diagnóstico operatorio de apendicitis (\%) \\
\hline Normal $^{*}$ & $27(62,8)$ & 15 & $11(40,7)$ \\
Líquido libre $^{\dagger}$ & $6(13,9)$ & 4 & $1(16,6)$ \\
Patología ginecológica $^{\ddagger}$ & $4(9,3)$ & 2 & $1(25)$ \\
Apendicitis aguda & $2(4,6)$ & 2 & $1(50)$ \\
Alteración intestinal & $2(4,6)$ & 2 & $2(100)$ \\
Absceso pericecal & $1(2,3)$ & 1 & $1(100)$ \\
Dilatación pielocalicial & $1(2,3)$ & 0 & - \\
\hline
\end{tabular}

*Un informe visualiza apéndice normal; $\uparrow$ en fondo de saco de Douglas, fosa ilíaca derecha (FID) y/o pericólico; $\ddagger$ dos quistes de ovario, un útero grávido, uno no especifica; $\S$ asas distendidas en FID con peristaltismo disminuido, engrosamiento parietal del colon. 


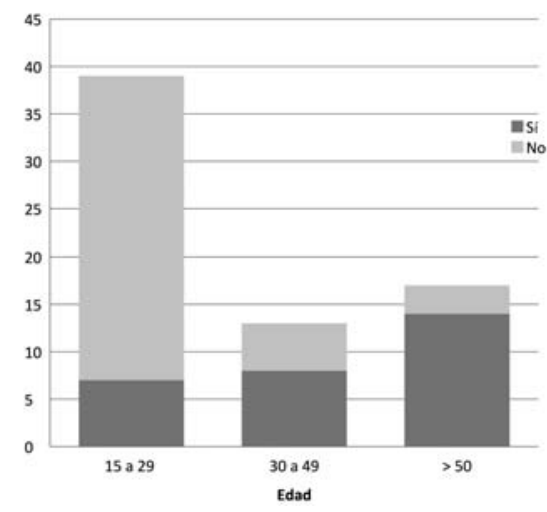

Figura 1. Solicitud de tomografía según rango de edad.

paroscópico, confirmando el diagnóstico en 50 casos (91\%), realizando la apendicectomía laparoscópica. En los cinco pacientes restantes ( $9 \%$ ) los hallazgos fueron: ulcus gastroduodenal perforado, folículo roto, adenitis mesentérica, diverticulitis y una laparoscopía en blanco. A 12 pacientes $(17,6 \%)$ se les realizó apendicetomía convencional, de los cuales un paciente presentó apéndice normal. En un caso, a pesar de que el diagnóstico clínico primario fue apendicitis aguda, se confirmó una enfermedad inflamatoria pélvica y no se realizó ningún procedimiento quirúrgico.

\section{Otros diagnósticos clínicos primarios}

De los 22 pacientes (13\%) que al inicio se planteó otro diagnóstico diferente al de apendicitis aguda o duda
Tabla 4. Características del dolor y síntomas acompañantes en los casos de planteo primario de apendicitis aguda.

\begin{tabular}{lc}
\hline Características del dolor & Número $(\%) n: 68$ \\
\hline Esquema de Murphy & $37(54,4)$ \\
Dolor de inicio en FID & $22(32,3)$ \\
Dolor en hemiabdomen inferior & $6(8,8)$ \\
Dolor difuso & $3(4,4)$ \\
\hline Síntomas acompañantes & \\
\hline Náuseas y vómitos & $50(73,5)$ \\
Anorexia & $34(50,0)$ \\
Fiebre & $19(27,9)$ \\
Síntomas urinarios & $3(4,4)$ \\
Deposiciones líquidas & $9(13,2)$ \\
\hline
\end{tabular}

FID: fosa ilíaca derecha

diagnóstica, a 7 pacientes (32\%) se les realizó una $\mathrm{LD}$, confirmando cinco casos de apendicitis, uno patología ginecológica y uno en blanco. En dos de los pacientes con apendicitis aguda se solicitó previamente una TC que no informó el diagnóstico.

Los 15 pacientes restantes (68\%) fueron dados de alta, derivados a otra especialidad o quedaron en observación sin realizar intervención quirúrgica.

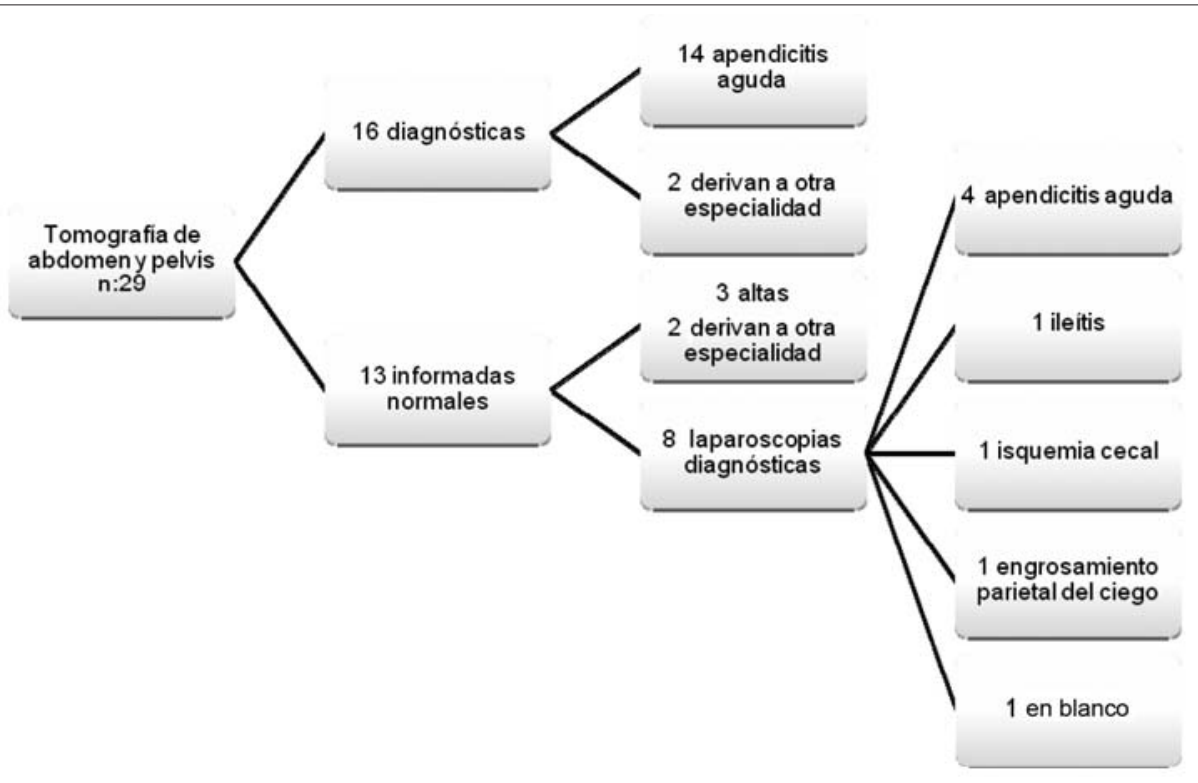

Figura 2. Informes tomográficos y resolución del paciente. 


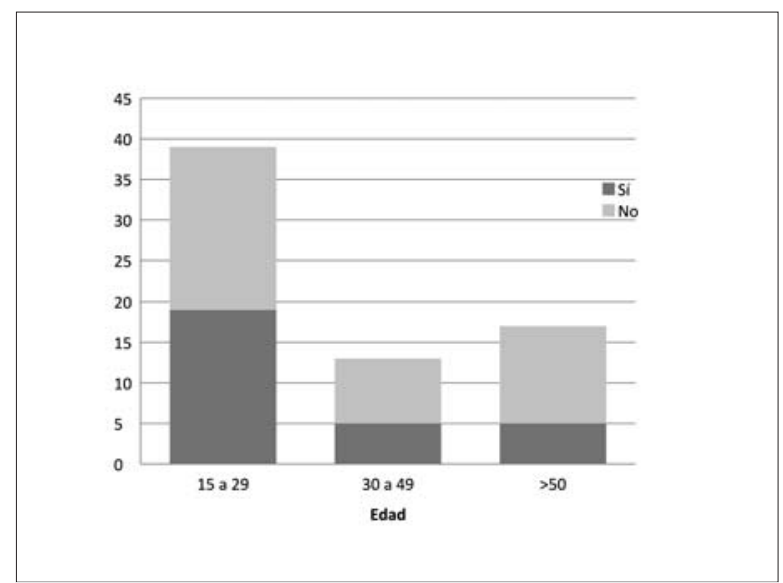

Figura 3. Laparoscopía diagnóstica según rango de edad.

\section{Diagnósticos definitivos}

De los 159 pacientes de nuestro estudio, el diagnóstico final de apendicitis aguda se realizó en 96 casos $(60,3 \%)$. En 32 pacientes (20\%) no se llegó a un diagnóstico definitivo, a algunos se les otorgó el alta luego de la valoración inicial, otros permanecieron en observación con mejoría de los síntomas o se les realizó una laparoscopía que fue en blanco con posterior alta. En 17 pacientes (11\%) el diagnóstico fue patología ginecológica, $8(5 \%)$ otros diagnósticos y 6 pacientes $(4 \%)$ patología urológica.

\section{Sensibilidad y especificidad de los estudios de imagen}

La sensibilidad y especificidad de la ecografía de abdomen en nuestro estudio para el diagnóstico de apendicitis fue de $19,4 \%$ IC al $95 \%:(5,3 ; 34,7 \%)$ y de $97,3 \%$ IC al $95 \%$ : $(90,9 ; 100 \%)$ respectivamente, con un valor predictivo positivo (VVP) de 87,5\% IC al 95\%: (58,3; $100 \%$ ) y un valor predictivo negativo (VPN) de $57,5 \%$ IC al 95\%: (44,1; 69,7\%).

La TC presentó una sensibilidad de $70 \%$ IC al 95\%: (47,4; 92,6\%) y especificidad de 100\% IC al 95\%: $(95,8$; $100 \%$ ) para el diagnóstico de apendicitis aguda, con un VPP $100 \%(96,4 ; 100 \%)$ y VPN $66,7 \%(42,1 ; 91,2 \%)$.

\section{Discusión}

Las consultas en los servicios de emergencia por cuadros de dolor a nivel de FID son comunes, a su vez los diagnósticos diferenciales son variados y dependen de la edad y sexo del paciente. Pueden abarcar afecciones digestivas, genitales, urinarias, osteomusculares, circulatorias y parietales. Dentro de los cuadros quirúrgicos la apendicitis aguda es el más frecuente, siendo el diagnóstico diferencial de mayor interés para el cirujano general. Los errores de diagnóstico conllevan a cirugías

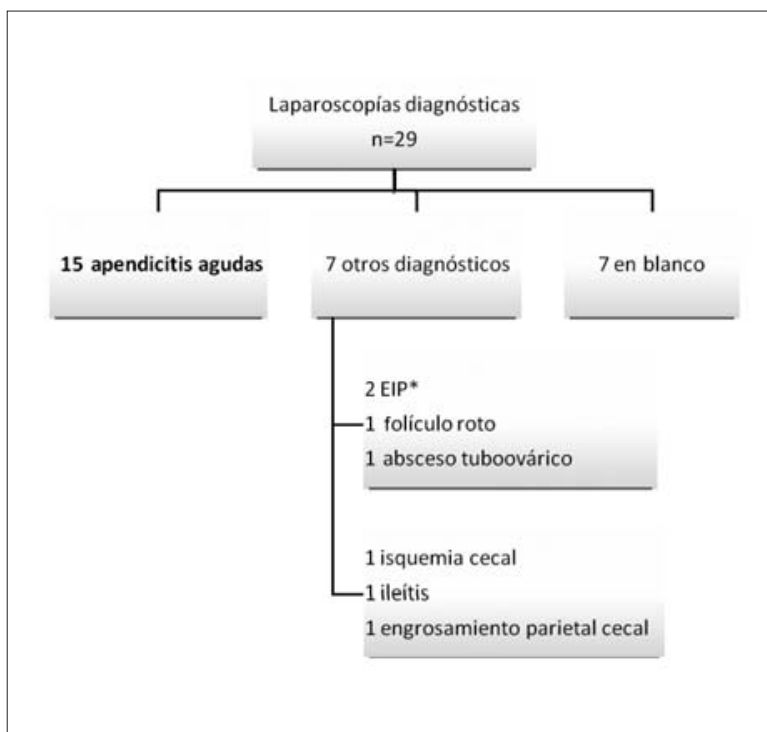

Figura 4. Laparoscopías diagnósticas realizadas y hallazgos intraoperatorios. *Enfermedad inflamatoria

Tabla 5. Hallazgos más frecuentes en el examen físico en los pacientes con planteo primario de apendicitis aguda.

\begin{tabular}{lc}
\hline $\begin{array}{l}\text { Hallazgos más frecuentes al } \\
\text { examen fisico }\end{array}$ & Número (\%) \\
\hline Dolor a la decompresión de FID & $38(55,8)$ \\
Defensa en FID & $28(41,2)$ \\
Dolor solo a compresión de FID & $10(14,7)$ \\
Tumoración palpable & $2(2,9)$ \\
\hline FID: fosa ilíaca derecha. & \\
\hline
\end{tabular}

innecesarias con apendicectomías negativas o a un diagnóstico tardío que conduce a perforaciones. Se entiende por apendicectomía negativa cuando los pacientes presentan datos clínicos de apendicitis aguda pero el análisis histopatológico posoperatorio del apéndice cecal es normal.

La estrategia diagnóstica debe comenzar siempre con un correcto interrogatorio y examen físico. La mayor incidencia de apendicitis aguda se encuentra entre la segunda y tercera década de la vida, por lo tanto es esperable que en nuestra población el pico máximo de edad se haya presentado entre los 17 y 18 años, si bien no hubo una importante diferencia en cuanto al sexo, con una leve tendencia hacia el femenino (53\%).

Luego de la valoración primaria realizada por un residente de cirugía general mediante anamnesis y examen físico, apoyado en paraclínica básica si lo conside- 
raba necesario (hemograma, examen de orina), se realizó diagnóstico primario de apendicitis aguda en el $43 \%$ de los pacientes, y en igual porcentaje se planteó duda diagnóstica. Como es de esperar, en la población con duda diagnóstica se incrementó el porcentaje de pacientes de sexo femenino a $67 \%$. Esto se debe al mayor número de diagnósticos diferenciales que se presentan en la mujer en edad reproductiva.

Con el fin de determinar o excluir otros diagnósticos, o para prevenir operaciones innecesarias, la utilización de los estudios de imagen en estos pacientes se ha incrementado con el paso de los años. En la literatura existen múltiples estudios para determinar su rol en el algoritmo diagnóstico del dolor en FID $^{(3-9)}$. La ecografía de abdomen y la TC son las más utilizadas. En un metaanálisis tanto la ecografía como la TC fueron altamente específicas (entre $93 \%$ y $95 \%$ ) en niños y adultos, mientras que la TC fue más sensible que la ecografía ( $94 \%$ vs $83 \%$ respectivamente) ${ }^{(4)}$.

En nuestro estudio, 27 (63\%) de las ecografías de abdomen de los pacientes en los cuales se planteó duda diagnóstica fueron informadas normales; de estas, 11 (41\%) presentaron diagnóstico final de apendicitis aguda. En solo 2 pacientes $(4,6 \%)$ se informó un apéndice patológico, confirmándose en el intraoperatorio en un solo caso.

La ecografía es una técnica no invasiva que no expone al paciente a irradiación, puede diagnosticar otras patologías causantes del cuadro, tales como la adenitis mesentérica, patología ginecológica o urológica, pero presenta la desventaja de ser fuertemente dependiente del operador $^{(3)}$.

En cuanto a la TC, Lee ${ }^{(5)}$ comparó una estrategia de TC obligatoria versus selectiva en pacientes con sospecha de apendicitis y menos de 72 horas de inicio de los síntomas. Hubo menos apendicectomías negativas $(2,6 \%$ vs $13,9 \%)$ y menos perforaciones $(10,3 \%$ vs $18,4 \%$ ) en el grupo sometido a TC obligatorios, aunque los resultados no fueron estadísticamente significativos. $\mathrm{Krajewski}^{(6)}$, en un metaanálisis que incluyó 28 estudios relacionados con el uso de la TC abdominal en el diagnóstico de apendicitis aguda, concluyó que la TC preoperatoria dio como resultado una reducción en la tasa de apendicectomías negativas, con un aumento en el tiempo hasta la cirugía, aunque sin aumento en la tasa de perforación.

Otros autores, como Gammeri ${ }^{(7)}$, consideran que la solicitud de TC tiene mayor valor en pacientes de más de 50 años, los cuales presentan riesgo aumentado de presentar otra patología distinta a apendicitis, como diverticulitis o cáncer de colon, las cuales deberían ser identificadas en el preoperatorio. Concluye que si no existen contraindicaciones, la TC debería ser mandatoria en los mayores de 50 años que se presentan con dolor en FID. La laparoscopía de emergencia en estos casos sería inapropiada y podría potencialmente ocasionar mayor daño que beneficio.

En nuestro trabajo, la TC se realizó a $32(20 \%)$ del total de pacientes que consultaron con dolor en FID, la mayoría comprendida en los pacientes que presentaban duda diagnóstica (29 de 32). De estas, la mitad aproximadamente fue en pacientes mayores de 50 años (a 78\% de los mayores de 50 años se le realizó TC vs $18 \%$ de los pacientes comprendidos entre 15 y 29 años, figura 1).

Destacamos que el total de las TC informadas como apendicitis aguda se confirmaron en el intraoperatorio. Por otro lado, $45 \%$ se informaron normales. En ocho pacientes se prosiguió con estudios diagnósticos y se les realizó una $\mathrm{LD}$, en la mitad se confirmó una apendicitis aguda, incluso uno presentaba un plastrón abscedado no informado en la TC.

El error diagnóstico en los informes tomográficos es un fenómeno mundial en creciente aumento. Según Howlett ${ }^{(8)}$, la discrepancia en los informes radiológicos de las TC es un tema complejo, las tasas publicadas de discrepancia para la TC abdominal varía ampliamente desde $<0,1 \%$ a $18 \%$. Las causas son numerosas, bien reconocidas y a menudo interrelacionadas. Las específicas de los radiólogos incluyen el razonamiento defectuoso, la falta de conocimiento, fracaso en la percepción o mala comunicación de los hallazgos. Los factores relacionados con el sistema son también importantes y se reconocen una serie de causas: personal joven, combinado con exceso de trabajo, inexperiencia del personal e información clínica o radiológica previa insuficiente o inexacta. La estrecha colaboración en persona entre el radiólogo y el equipo quirúrgico está asociada con cambios significativos y frecuentes en el manejo del paciente, incluso cuando el informe radiológico es correcto y contiene la información diagnóstica necesaria ${ }^{(8)}$.

Aunque la exactitud diagnóstica de los estudios radiológicos es muy alta en la literatura publicada, es importante dentro de cada institución evaluar su utilidad "real". En un estudio realizado por Cuschieri ${ }^{(9)}$, la variación de apendicectomías negativas entre hospitales estuvo estrechamente relacionada con la exactitud del informe TC y ecográfico, y sugiere que su precisión debe considerarse una medida de calidad en el cuidado de pacientes con presunta apendicitis.

Por otro lado, las ventajas de la laparoscopía son mundialmente aceptadas para procedimientos electivos. Se ha demostrado su seguridad y beneficios también en pacientes con cuadros agudos abdominales. De hecho, el abordaje laparoscópico en la emergencia es capaz de proporcionar una mejor visión de toda la cavidad abdominal con un trauma mínimo dando la posibilidad de 
realizar un diagnóstico preciso y al mismo tiempo un tratamiento definitivo ${ }^{(10)}$. Las ventajas de la cirugía miniinvasiva son particularmente atractivas en la emergencia y su uso ha ido en aumento en los últimos diez años ${ }^{(10,11)}$.

Se ha demostrado un alto rendimiento diagnóstico a favor de la LD entre el $90 \%$ y $100 \%$, comparando grupos de pacientes con LD versus observación ${ }^{(1,2,12)}$. Asimismo, se ha constatado un cambio en la estrategia terapéutica con la realización de la LD en hasta un $60 \%$ de $\operatorname{casos}^{(1)}$. Dos metaanálisis comparan el uso de la laparoscopía temprana versus observación en pacientes con dolor abdominal agudo. Analizaron distintas variables, solo un parámetro (porcentaje de pacientes con diagnóstico incierto) fue significativamente mejorado con la realización de la LD. Los tres restantes parámetros: estadía hospitalaria, complicaciones y reingresos, fueron al menos 50\% menores en el grupo de la LD, pero sin valor estadístico significativo. La falta de información uniforme y la heterogeneidad de los distintos estudios no permiten establecer como recomendación de rutina el uso de LD temprana en pacientes con dolor abdominal agudo inespecífico. Sin embargo, afirman que no existe evidencia de que el uso de la misma sea perjudicial ${ }^{(13,14)}$.

En nuestra serie, de los 69 pacientes con duda diagnóstica inicial, se les realizó una LD a 29 pacientes (42\%) (8 tenían TC informada normal). En el $52 \%$ se diagnosticó apendicitis aguda, realizando su tratamiento definitivo en el mismo acto quirúrgico; $24 \%$ realizaron diagnósticos diferenciales, y otro $24 \%$ fueron normales culminando el procedimiento. Si bien no presentamos complicaciones relacionadas con el procedimiento en sí, seguramente por el bajo número de pacientes, destacamos que no está exento de morbimortalidad.

En un estudio prospectivo realizado por Moberg ${ }^{(15)}$ en el que se realizaron 1.043 LD por sospecha de apendicitis aguda, ninguno de los 211 pacientes en los que se realizó una LD como único proceder fue readmitido para realizar la apendicectomía durante el seguimiento posoperatorio (dos años). La tasa de complicaciones fue de $2 \%$ en el grupo de LD y de $10 \%$ en el que se realizó apendicectomía. Concluye que la LD como único proceder es un procedimiento seguro. Lee ${ }^{(16)}$, en un estudio retrospectivo, afirma que la extracción de un apéndice macroscópicamente normal tiene una morbilidad similar a la extracción de un apéndice inflamado, desaconsejando su extracción rutinaria en la LD.

Según las pautas de la Society of American Gastrointestinal and Endoscopic Surgeons ${ }^{(1)}$, las indicaciones para el uso de la laparoscopía en el dolor abdominal agudo son: dolor abdominal de causa incierta de menos de siete días de duración luego de realizar exámenes iniciales o como alternativa a la observación en pacientes con dolor abdominal inespecífico. Las contraindicaciones absolutas son inestabilidad hemodinámica, coagulopatía no corregida y aquellos procesos fisiopatológicos que no toleran el neumoperitoneo. Las contraindicaciones relativas son cirugías abdominales previas, dolor crónico, obesidad, embarazo e hipertensión portal. Los riesgos potenciales son la demora en el tratamiento definitivo con mayor morbimortalidad cuando el estudio es falso negativo y complicaciones relacionadas al procedimiento y anestesia ${ }^{(1)}$. Los beneficios son la reducción de las tasas de laparotomías negativas con subsecuente menor estadía hospitalaria, menor morbilidad y menor costo, diagnóstico temprano y posibilidad de tratamiento miniinvasivo con mejores resultados comparado con la observación ${ }^{(1)}$.

Las ventajas de la LD en mujeres en edad reproductiva, con dolor abdominal inespecífico o sospecha de apendicitis, incluyen una mayor tasa de diagnósticos específicos y una menor tasa de apendicectomías negativas en comparación con la apendicectomía convencional y menor estadía hospitalaria ${ }^{(17,18)}$. Sin embargo, otros estudios sostienen que el aumento del uso de la cirugía laparoscópica ha producido un incremento en el número de cirugías innecesarias, principalmente en mujeres, para el manejo del dolor en FID. Por lo que debería sopesarse el riesgo asociado a una cirugía sobre la necesidad de obtener un diagnóstico ${ }^{(19,20)}$.

La literatura disponible tiene una serie de limitaciones, incluida la falta de homogeneidad en las poblaciones de pacientes y la ausencia frecuente de estudios de imágenes preoperatorios de alta calidad, que podrían haber proporcionado el diagnóstico sin la necesidad de un procedimiento invasivo. Además, se necesitan estudios de investigación de mejor calidad para evaluar el papel definitivo de la $\mathrm{LD}$ en pacientes con dolor abdominal de causa inespecífica ${ }^{(2)}$.

\section{Sesgos}

Existe un porcentaje desconocido de paciente es que consultaron con dolor en FID que no ingresaron a nuestro estudio principalmente por dos motivos, el primero es que algunos de ellos fueron valorados por el médico del servicio de urgencia y dados de alta sin realizar la consulta con el cirujano de guardia; el segundo motivo es que no estuvieron cubiertos todos los días de la semana del período correspondiente, ya sea porque no había residente de guardia o porque este no quiso participar del estudio.

Otro sesgo es la falta de seguimiento de los pacientes (20\%) que fueron dados de alta, permanecieron en observación o se les realizó una laparoscopía en blanco, ya que desconocemos si reconsultaron por dolor o si alguno requirió posteriormente ser reintervenido. 


\section{Conclusiones}

La LD fue un procedimiento seguro en nuestro estudio y permitió realizar el tratamiento de la patología que presentaba el paciente en la mayoría de los casos, si bien sabemos que no está exenta de posibles complicaciones.

La TC fue solicitada de forma selectiva para el estudio de pacientes con dolor en FID, casi exclusivamente en aquellos pacientes con diagnóstico incierto y estando en estrecha relación con la edad del paciente. A su vez, si bien tiene una buena especificidad para diagnosticar apendicitis aguda, su sensibilidad en nuestro estudio fue baja en comparación con datos internacionales.

El algoritmo diagnóstico debe ser individualizado para cada paciente, basado en protocolos adaptados a los recursos con los que se dispone en cada institución.

\section{Agradecimiento}

A Alejandro Noria por su colaboración en el análisis estadístico de los datos.

\section{Abstract}

Introduction: right iliac fossa (RIF) pain is a frequent reason for consultation at the Emergency Units. The main challenge lies in defining whether it is a surgical condition or not.

Objective: to learn about the role of diagnostic laparoscopy, abdominal ultrasound and CT scan in the studies algorithm of patients with RIF pain of unknown etiology in Uruguay.

Method: observational, descriptive, prospective, multi-center study of patients who consulted for RIF from June 1st to August 31, 2017 in Health Institutions in Uruguay. The following data were gathered in an excel sheet: sex, age, symptoms, physical findings, tests requested, conduct adopted, intraoperative findings and complications.

Results: 159 sheets were completed. Upon a primary assessment a diagnostic doubt was arose in 69 patients $(43.4 \%)$. A CT scan was requested to 29 patients, and a CT was performed to $78 \%$ of patients older than 50 years old vs $18 \%$ of those between 15 and 29 years

Conclusions: the diagnostic laparoscopy allowed for diagnosis in most cases. Sensitivity of the CT scan was low when compared to international data. The diagnostic algorithm needs to me individualized, based on protocols adapted to the resources of every institution.

\section{Resumo}

Introdução: a dor na fossa ilíaca direita (FID) é um motivo habitual de consulta habitual nos Serviços de Urgências. O principal desafio é diferenciar entre um quadro cirúrgico e um não cirúrgico.
Objetivo: conhecer o papel da laparoscopia diagnóstica (LD), do ultrassom de abdômen e da tomografia computada (TC) no algoritmo de estudo dos pacientes com dor na FID com diagnóstico incerto no Uruguai.

Método: estudo observacional, descritivo, prospectivo, multicêntrico sobre pacientes que consultaram por dor na FID no período $1^{\circ}$ de junho - 31 de agosto del 2017 em Centros Assistenciais no Uruguai. Em una planilha electrónica foram incluídos os seguintes dados: sexo, idade, sintomatologia, achados físicos, exames solicitados, conduta tomada, achados intra-operatórios e complicações.

Resultados: foram preenchidas 159 planilhas. Depois de uma avaliação primaria foram definidas dúvidas diagnósticas em 69 (43,4\%) pacientes. Foram solicitadas TC a 29 (42\%) pacientes; 78\% dos pacientes com mais de 50 anos vs $18 \%$ dos pacientes com idades entre 15 e 29 anos. 13 (44,8\% do total) tiveram laudo "normal", confirmando apendicite aguda em $4(30,7 \%)$ durante cirurgia. Foram realizadas 29 LD nos casos com dúvida diagnóstica sem dos terços em pacientes de 15 a 29 anos. Em 22 (76\%) casos foi possível definir um diagnóstico. Não se registraram complicações.

Conclusões: a LD permitiu realizar diagnóstico e tratamento na maioria dos casos. A sensibilidade da TC foi baixa em comparação com dados internacionais. $\mathrm{O}$ algoritmo diagnóstico deve ser individualizado, baseado em protocolos adaptados aos recursos de cada instituição.

\section{Bibliografía}

1. Hori Y; SAGES Guidelines Committee. Diagnostic laparoscopy guidelines: this guideline was prepared by the SAGES Guidelines Committee and reviewed and approved by the Board of Governors of the Society of American Gastrointestinal and Endoscopic Surgeons (SAGES), November 2007. Surg Endosc 2008; 22(5):1353-83.

2. Agresta F, Ansaloni L, Baiocchi GL, Bergamini C, Campanile FC, Carlucci M, et al. Laparoscopic approach to acute abdomen from the Consensus Development Conference of the Società Italiana di Chirurgia Endoscopica e nuove tecnologie (SICE), Associazione Chirurghi Ospedalieri Italiani (ACOI), Società Italiana di Chirurgia (SIC), Società Italiana di Chirurgia d'Urgenza e del Trauma (SICUT), Società Italiana di Chirurgia nell'Ospedalità Privata (SICOP), and the European Association for Endoscopic Surgery (EAES). Surg Endosc 2012; 26(8):2134-64.

3. Wray CJ, Kao LS, Millas SG, Tsao K, Ko TC. Acute appendicitis: controversies in diagnosis and management. Curr Probl Surg 2013; 50(2):54-86.

4. Doria AS, Moineddin R, Kellenberger CJ, Epelman M, Beyene J, Schuh S, et al. US or CT for Diagnosis of appendicitis in children and adults?: a Meta-Analysis. Radiology 2006; 241(1):83-94. 
5. Lee CC, Golub R, Singer AJ, Cantu R Jr, Levinson H. Routine versus selective abdominal computed tomography scan in the evaluation of right lower quadrant pain: a randomized controlled trial. Acad Emerg Med 2007; 14(2):117-22.

6. Krajewski S, Brown J, Phang PT, Raval M, Brown CJ. Impact of computed tomography of the abdomen on clinical outcomes in patients with acute right lower quadrant pain: a meta-analysis. Can J Surg 2011; 54(1):43-53.

7. Gammeri E, Catton A, van Duren BH, Appleton SG, van Boxel GI. Towards an evidence-based management of right iliac fossa pain in the over 50-year-old patient. Ann R Coll Surg Engl 2016; 98(7):496-9.

8. Howlett DC, Drinkwater K, Frost C, Higginson A, Ball C, Maskell G. The accuracy of interpretation of emergency abdominal CT in adult patients who present with non-traumatic abdominal pain: results of a UK national audit. Clin Radiol 2017; 72(1):41-51.

9. Cuschieri J, Florence M, Flum DR, Jurkovich GJ, Lin P, Steele SR, et al. Negative appendectomy and imaging accuracy in the Washington State Surgical Care and Outcomes Assessment Program. Ann Surg 2008; 248(4):557-63.

10. Agresta F, Campanile FC, Podda M, Cillara N, Pernazza G, Giaccaglia V, et al. Current status of laparoscopy for acute abdomen in Italy: a critical appraisal of 2012 clinical guidelines from two consecutive nationwide surveys with analysis of 271,323 cases over 5 years. Surg Endosc 2017; 31(4):1785-95.

11. McCartan DP, Fleming FJ, Hill AD. Patient and surgeon factors are associated with the use of laparoscopy in appendicitis. Colorectal Dis 2012; 14(2):243-9.

12. Golash V, Willson PD. Early laparoscopy as a routine procedure in the management of acute abdominal pain: a review of 1,320 patients. Surg Endosc 2005; 19(7):882-5.
13. Maggio AQ, Reece-Smith AM, Tang TY, Sadat U, Walsh SR. Early laparoscopy versus active observation in acute abdominal pain: systematic review and meta-analysis. Int J Surg 2008; 6(5):400-3.

14. Domínguez LC, Sanabria A, Vega V, Osorio C. Early laparoscopy for the evaluation of nonspecific abdominal pain: a critical appraisal of the evidence. Surg Endosc 2011; 25(1):10-8.

15. Moberg AC, Ahlberg G, Leijonmarck CE, Montgomery A, Reiertsen O, Rosseland AR, et al. Diagnostic laparoscopy in 1043 patients with suspected acute appendicitis. Eur J Surg 1998; 164(11):833-40

16. Lee M, Paavana T, Mazari F, Wilson TR. The morbidity of negative appendicectomy. Ann R Coll Surg Engl 2014; 96(7):517-20.

17. Garbarino S, Shimi SM. Routine diagnostic laparoscopy reduces the rate of unnecessary appendicectomies in young women. Surg Endosc 2009; 23(3):527-33.

18. Gaitán HG, Reveiz L, Farquhar C, Elias VM. Laparoscopy for the management of acute lower abdominal pain in women of childbearing age. Cochrane Database Syst Rev 2014;(5):CD007683.

19. Jones GE, Kreckler S, Shah A, Stechman MJ, Handa A. Increased use of laparoscopy in acute right iliac fossa pain - is it good for patients? Colorectal Dis 2012; 14(2):237-42.

20. McGreevy JM, Finlayson SR, Alvarado R, Laycock WS, Birkmeyer CM, Birkmeyer JD. Laparoscopy may be lowering the threshold to operate on patients with suspected appendicitis. Surg Endosc 2002; 16(7):1046-9. 